\title{
Study of the smartphone operation support application for visually impaired
}

\author{
Taiki Torigoe ${ }^{\mathrm{a}, *}$, Yoshihisa Nakatoh ${ }^{\mathrm{a}}$ \\ ${ }^{a}$ Kyushu Institute of Technology \\ 1-1, Sensui-cho, Tobata-ku, Kitakyusyu-shi, Fukuoka, 804-0015, Japan \\ *q349421t@mail.kyutech.jp
}

\begin{abstract}
Currently, smartphones are rapidly spreading, and a convenient daily life. So, smartphone is indispensable for many people, and no exception for visually impaired people. However, the penetration rate of smartphones to visually impaired people is low. It is difficult to grasp the complicated screen configuration. Therefore, it takes a very long time to perform the operation. In order to support the operation of a smartphone, we created the touch screen application for visually impaired people to get the information easily. Proposed method guides the user to the application that the user actually want to use. As a result of comparing the proposed method with the conventional method, we confirmed a drastic reduction in operation time of the proposed method.
\end{abstract}

Keywords: Operation Support System, Visually Impaired, Smartphone, application.

\section{Introduction}

Currently, smartphones are rapidly spreading, and a convenient daily life. Therefore, smartphone is indispensable for many people, and visually impaired people are no exception. In fact, visually impaired people feel smartphones are more convenient than mobile phones and think that visually impaired people want to use smartphones. However, the penetration rate of smartphones to visually impaired people is low ${ }^{(1)}$. Usually, when visually impaired people uses smartphones, the people grasp the operation statue from auditory information obtained by the screen reader and grasps the relative position on the touch screen through the tactile information. This operation method is difficult for visually impaired people to grasp complicated screen configuration. Therefore, visually impaired people takes very long time to operate the smartphone. In addition, There are many disadvantages for visually impaired which are "visually impaired has few teaching materials to learn how to operate the touch screen device, and the seminars or workshops are not enough", "few applications are made with consideration for visually impaired people", "the screen layout and structure of the smartphone is too complicated", so it is considered that the utilization of rate is low ${ }^{(2)}$.

In this research, in order to support the operation of smartphone, we created the touch screen application for visually impaired people to get the information easily. As for the operation of the system, after hearing the operation guidance by voice, the user always inputs characters in the same layout and the user continues to select words to achieve the goal. And we examined this operation support application.

\section{Overall Structure of Operation Support Application}

Figure 1 is screenshot of the proposed application. The proposed application is composed of the speech synthesizing part for speech synthesizing contents desired by the user to operate, the input part that the user inputs characters, and the predictive transformation part that performs predictive transformation based on the input character. This time, the proposed application is running on the iPhone, the speech synthesizer uses Siri, the input part uses the software keyboard, and the top six words of predictive transformation are displayed. 


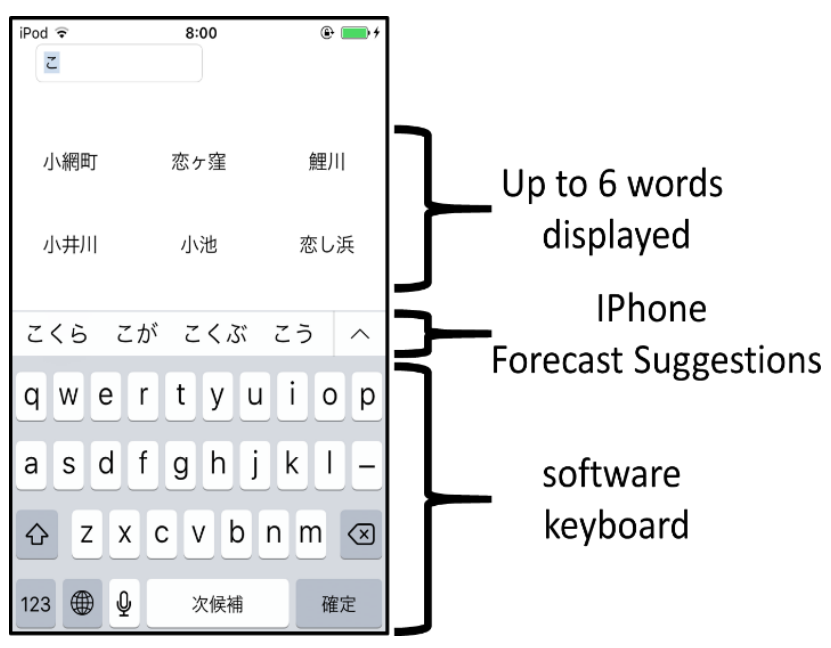

FIG.1. Screenshot of the proposed application

\section{Details of smartphone operation support system}

Various smartphone applications exist, but basically smartphones are being developed assuming that healthy people directly touches the screen and performs operation. Although various smartphone applications exist, basically the smartphone is developed assuming that healthy people performs touch operation while viewing the screen directly. Therefore, in order to realize easy-to-use operation for the visually impaired people, consider inputting information using the touch screen application that the people are easy to input.

\subsection{Proposed application concept}

Figure 2 is the conceptual diagram showing the transfer guidance application as an example. It is possible to display the target page that the user is requesting by inputting characters such as the station name in the proposed application and passing the input information as data to the conventional transfer guide application. At this time, the user can always operate with the same layout even if the application want to be operated is different from the transfer guide application or the input information is different.

\subsection{Whole processing method of proposed application}

Figure 3 is processing block of the proposed application. The user inputs characters after hearing the operation guidance by voice synthesis, and selects the target word displayed by predictive conversion. Then, the next operation guidance is announced by voice synthesis, and the user continues to select the target word until the target

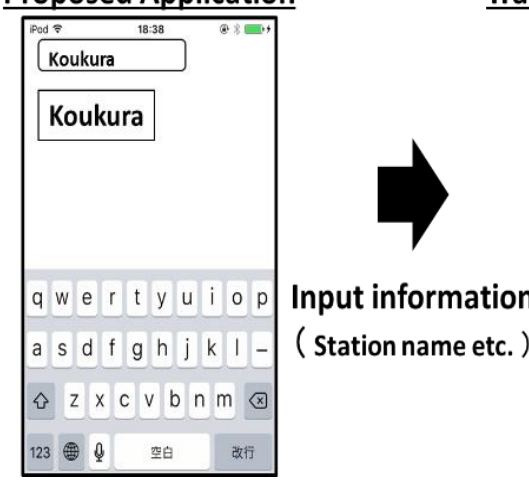

Transfer Guide Application

FIG.2. The conceptual diagram showing the transfer guidance application as an example

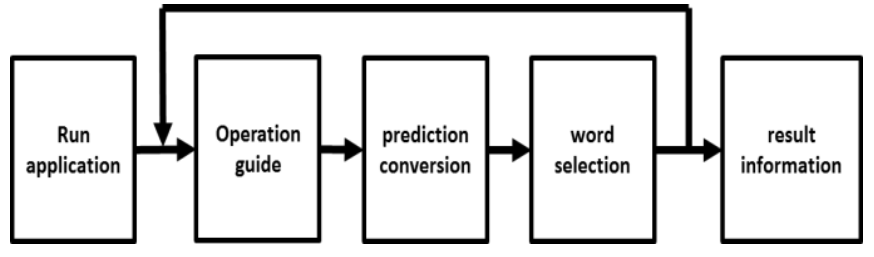

FIG.3. Processing block of the proposed application

page is displayed. Finally, the proposed application announces the result information at a speech speed slightly later than usual based on the selected word information.

\subsection{Details of predictive transformation}

Figure 4 is predictive transformation processing block. At this time, the word dictionary contains not only a character string but also part of speech information and frequency information. Therefore, if the user wants to input the application name, only the application name is selected as prediction candidates. The words rearranged in descending order of the frequency information are displayed on the screen of the smartphone. In addition, as a word selection support by character input, the top six words are announced by speech synthesis when the number of word candidates is 6 or less, and the number of candidates is announced when the number of word candidates is 7 or more.

\section{Experimental condition}

We compare the operation time with the conventional transfer guide application and the proposed application In order to verify the effectiveness of the proposed application. And we carry out the measurement after considering operation errors in order to simulate the actual operation situation. Figure 5 is the operation screen of the transfer 


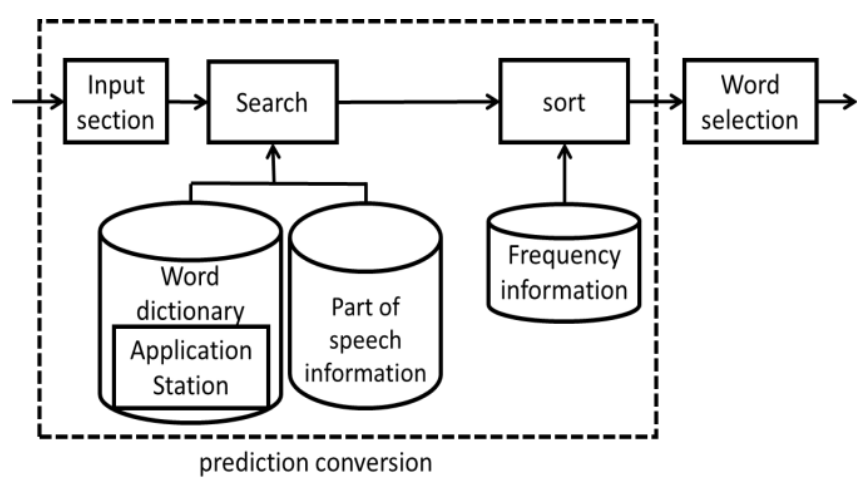

FIG.4. Predictive transformation processing block

guide application. (Example) We set "departure station: Kyushukoudaimae station, arrival station: Kokura station, time designation: 18 o'clock arrival", so that information such as departure time, arrival time, freight etc is obtained. The operation time of transfer guidance is (1)input of departure station and arrival station (2)time specification (3) acquisition of information, measured. We conduct experiments with five healthy people in their twenties as subjects and conduct experiments with 6 trials. Also, we use the iPhone 's VoiceOver and screen curtain to simulate visually impaired people' s operation and measure in three states of screen curtain OFF (screen display), ON (screen non-display), and eye mask. In measuring, we teach subjects how to use VoiceOver, and subjects understand the screen composition of both conventional application and proposed application. Also, the software keyboard use the QWERTY array. This is because the penetration rate of visually impaired people's PC is high, and it is used on a daily basis.

\section{Experimental condition}

Figure 6 is the result of the total time of operation for each trial. Focusing on the state of screencurtain ON / blindfold for the first time, it can be seen that the conventional method requires 1.99 times longer than the proposed method. This is because the conventional method is more complicated in the screen configuration than the proposed method. And the operation time become shorter for both the conventional method and the proposed method until the third time, and the fluctuation of the operation time become smaller after the fourth time because the user got used to the operation. Moreover, it turn out that the proposed method has shorter operation time than conventional method in most times.

Figures7, 8 and 9 are the results of three operation

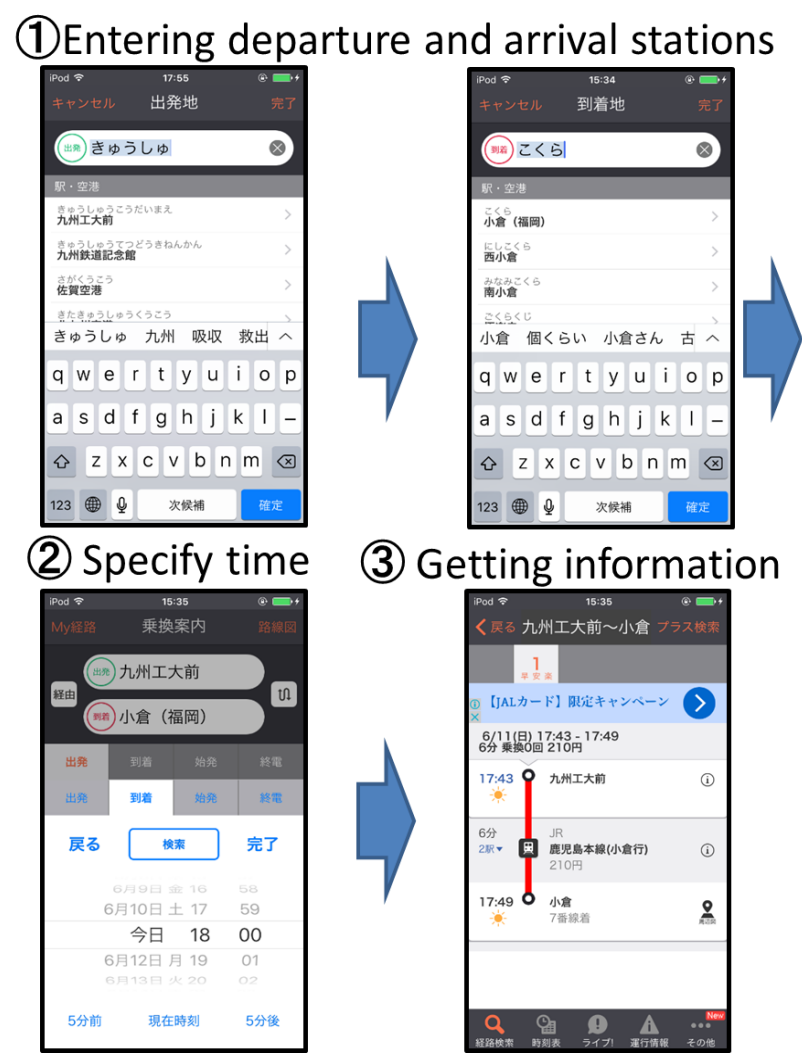

FIG.5. The result of the total time of operation for each trial

contents "station input", "Specify time" and " Getting information". Focusing on operations for the 4th or later time, when comparing the conventional method and the proposed method with the screen hiding / blindfold method, it took time "the station input: 1.1 times", "the time designation: 1.7 times". And there is almost no time lag in "obtaining information". "Time designation" :the time designation of the conventional method is picker, and picker operation with VoiceOver ON does not read numbers such as 1 or 2 . Therefore, the user has to make judgment only with small sounds that occur when options change, so it takes time to perform the operation. Also, with the time designation of the proposed method, if the user wants to set the time to 18 o'clock, the user needs to switch the keyboard to a number and enter "18: 00". So, we considered that operation is easier than conventional methods. "Station input / information acquisition": Unlike "time designation" there is no complicated operation. So, if the user grasps the screen composition, there is no difference in the operation method between the conventional method and the proposed method.

Figure10 is the diagram averaging total time of the fourth to sixth times. When comparing the conventional method and the proposed method with the screencurtain 


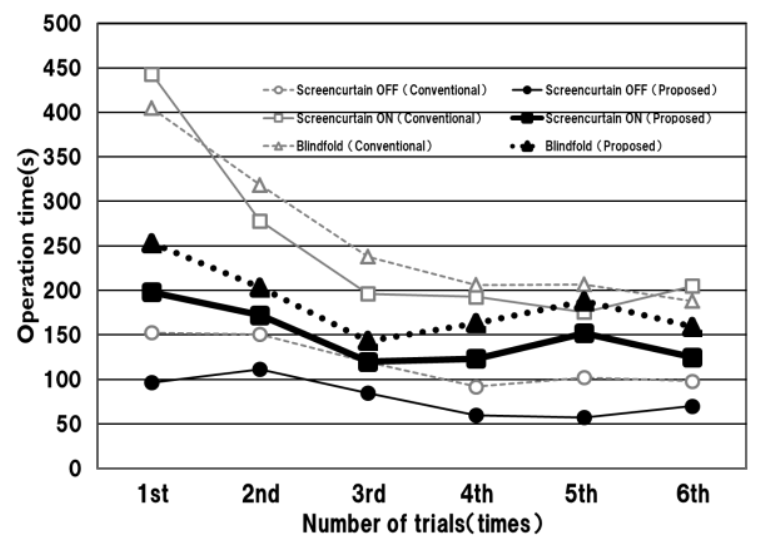

FIG.6. Result of the "total" time for each trial

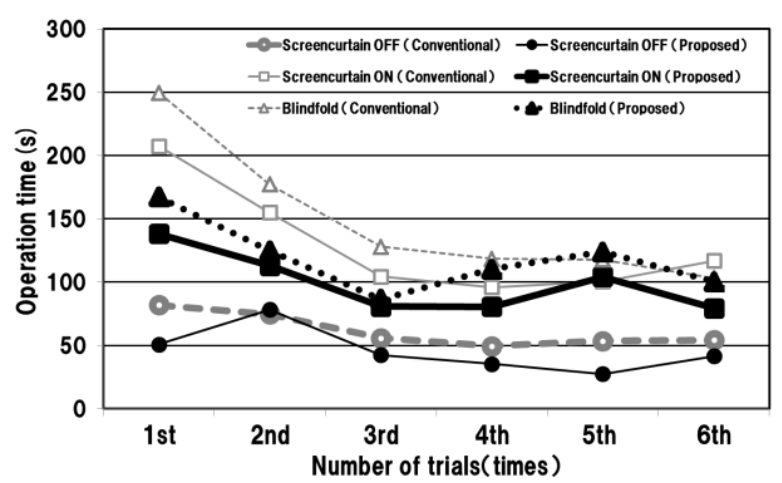

FIG.7. Result of the "station input" for each trial

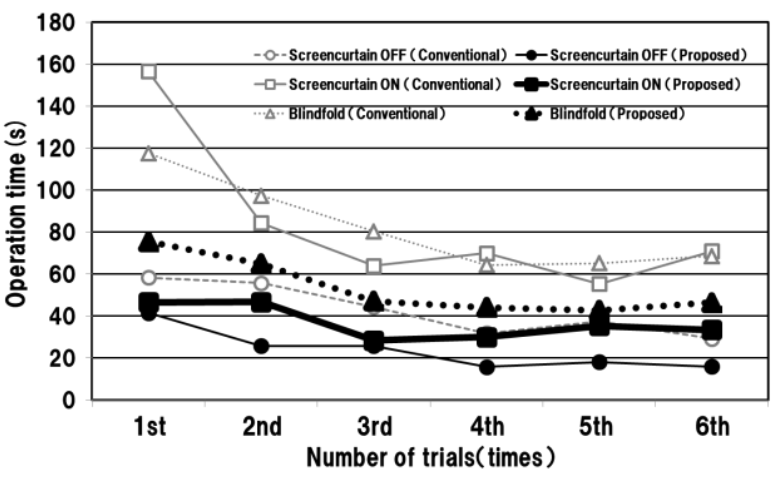

FIG.8. Result of the " time designation " for each trial

$\mathrm{ON}$ / blindfold state, we find that it is possible to operate in 0.7 times and 0.85 times time. Moreover, when comparing the screen display of the conventional method and the screen screencurtain $\mathrm{ON} /$ blindfold of the conventional method and the proposed method respectively, it is operation time the conventional method (screencurtain $\mathrm{ON})$ :1.96 times, the conventional method (blindfold): 2.06 times, the proposed method (screencurtain ON):1.37 times, the proposed method (blindfold): 1.74 times, and it was confirmed that the proposed method can shorten the operation time more than the conventional method even after accustoming to the operation.

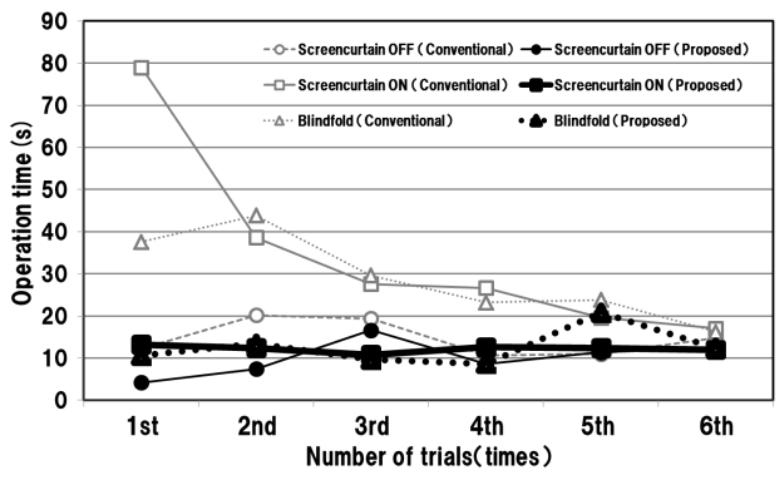

FIG.9. Result of the " Getting information" for each trial

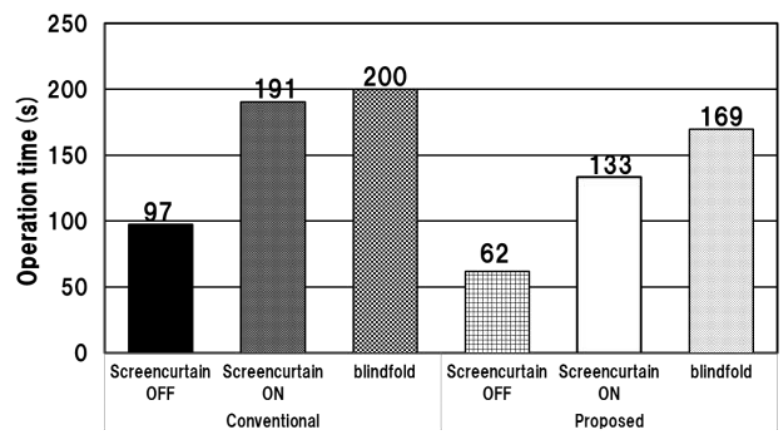

FIG.10. the diagram averaging total time of 4th to 6th times

\section{Conclusion}

This research proposed a new approach for visually impaired people to operate the smartphone. The proposed application is contributed shorten the operation time than the conventional transfer application which the effectiveness proposed application is provide. In the future, we plan to improve the operating ablity of the proposed application and to conduct a pratical experiments for visually impaired people.

\section{References}

(1) Tetsuya Watanabe and Toshimitsu Yamaguchi and Kanori Nanitani, Survey on the Use of Phones, Smartphones, Tablets and Personal Computers on the Visually Impaired, 2013, the Telecommunications Dissemination Foundation (2012), pp. 35-36,2014

(2) Haruo Matsuzaka, Study on Use of Touch Screen Terminal and User Interface for Visually Impaired, pp. 30-36, 2012. 